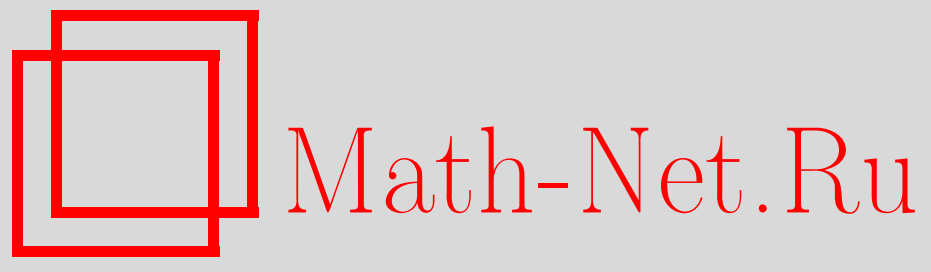

Н. Н. Осипов, Об асимптотике некоторых теоретикочисловых сумм, Матем. заметки, 2008, том 83, выпуск $3,472-476$

DOI: https://doi.org/10.4213/mzm4532

Использование Общероссийского математического портала Math-Net.Ru подразумевает, что вы прочитали и согласны с пользовательским соглашением http://www . mathnet.ru/rus/agreement

Параметры загрузки:

IP: 54.147 .182 .235

26 апреля 2023 г., 13:49:56

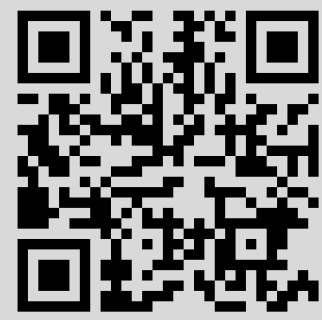




\section{Об асимптотике некоторых теоретико-числовых сумм}

\section{Н. Н. Осипов}

1. Введение. Пусть $\theta$ - плохо приближаемое вещественное иррациональное число, например, квадратичная иррациональность (определение и основные факты о плохо приближаемых числах см., например, в $[1 ; \S 5$ гл. I]). При $s>1$ хорошо известны (см., например, [2], [3]) следующие двусторонние оценки:

$$
\sigma_{1}(N, \theta, s)=\sum_{n=1}^{N} \frac{1}{\langle n \theta\rangle^{s}} \asymp N^{s}, \quad \sigma_{2}(N, \theta, s)=\sum_{n=1}^{N} \frac{1}{\langle n \theta\rangle^{s} n^{s}} \asymp \ln N
$$

(здесь и далее $\langle t\rangle$ обозначает расстояние от $t$ до ближайшего целого числа, а константы в оценках зависят, вообще говоря, от $\theta$ и $s$ ). Легко видеть, что предел отношения $\sigma_{1}(N, \theta, s) / N^{s}$ при $N \rightarrow \infty$ не существует (при $N=q_{k}$, где $q_{k}-$ знаменатель $k$-й подходящей дроби для числа $\theta$, это отношение терпит скачок). Вопрос о существовании предела отношения $\sigma_{2}(N, \theta, s) / \ln N$ является гораздо более трудным и не исследован, насколько известно автору, даже в случае, когда $\theta$ является квадратичной иррациональностью.

В настоящей заметке решается вопрос об асимптотике схожей с $\sigma_{2}(N, \theta, s)$ суммы

$$
\sum_{n=1}^{N} \frac{1}{\left(N^{1-s}+\langle n \theta\rangle^{s}\right) n^{s}}, \quad s>1,
$$

где $\theta$ - квадратичная иррациональность такая, что $\theta+\theta^{\prime} \in \mathbb{Z}$ (штрих означает переход к сопряженному числу). Решение этого вопроса основано на знании асимптотического поведения при $h \rightarrow 0$ сумм вида

$$
S(h, \mathrm{M}, s)=\sum_{(0,0) \neq(x, y) \in \mathrm{M}} \frac{1}{\left(h^{s}+|x|^{s}\right)\left(h^{s}+|y|^{s}\right)}, \quad s>1,
$$

где $\mathrm{M}$ - решетка в плоскости $\mathbb{R}^{2}$, изображающая какой-нибудь полный модуль в вещественном квадратичном поле (о методе и примерах получения асимптотических оценок подобных сумм см. [4]).

2. Основной результат. Пусть $\theta$ - вещественная квадратичная иррациональность. Рассмотрим в квадратичном поле $\mathfrak{K}=\mathbb{Q}(\theta)$ модуль

$$
\mathfrak{M}=\{1,-\theta\}=\left\{m-n \theta:(m, n) \in \mathbb{Z}^{2}\right\}
$$

и пусть $\mathrm{M}$ - решетка в плоскости $\mathbb{R}^{2}$, точки которой изображают числа из $\mathfrak{M}$ (о геометрическом изображении алгебраических чисел см., например, [5; §3 гл. II]).

Лемма. При $h \rightarrow 0$ справедлива оценка

$$
S(h, \mathrm{M}, s)=\frac{2}{\left|\theta-\theta^{\prime}\right|^{s}} \sum_{n \geqslant 1}\left(\frac{1}{\left(h^{s}+\langle n \theta\rangle^{s}\right) n^{s}}+\frac{1}{\left(h^{s}+\left\langle n \theta^{\prime}\right\rangle^{s}\right) n^{s}}\right)+O(1) .
$$

Работа выполнена при поддержке Российского фонда фундаментальных исследований (гранты №№ 06-01-00597, 07-01-00326). 
ДокАзАТЕЛЬСтво. Для целого $n \neq 0$ пусть целые $m_{1}(n), m_{2}(n)$ определяются равенствами

$$
\left|m_{1}(n)-n \theta\right|=\langle n \theta\rangle, \quad\left|m_{2}(n)-n \theta^{\prime}\right|=\left\langle n \theta^{\prime}\right\rangle .
$$

Ясно, что $m_{1}(n) \neq m_{2}(n)$, если $n$ достаточно велико. Имеем

$$
\begin{aligned}
S(h, \mathrm{M}, s) & =\sum_{(0,0) \neq(m, n)} \frac{1}{\left(h^{s}+|m-n \theta|^{s}\right)\left(h^{s}+\left|m-n \theta^{\prime}\right|^{s}\right)} \\
& =\sum_{n=0, m \neq 0}+\sum_{n \neq 0, m=m_{1}(n)}+\sum_{n \neq 0, m=m_{2}(n)}+\sum_{n \neq 0, m \notin\left\{m_{1}(n), m_{2}(n)\right\}}+O(1) \\
& =S_{1}+S_{2}+S_{3}+S_{4}+O(1) .
\end{aligned}
$$

Очевидно, $S_{1}=O(1)$. Далее,

$$
S_{2}=\sum_{n \neq 0} \frac{1}{\left(h^{s}+\langle n \theta\rangle^{s}\right)\left(h^{s}+\left|m_{1}(n)-n \theta^{\prime}\right|^{s}\right)}=\frac{1}{\left|\theta-\theta^{\prime}\right|^{s}} \sum_{n \neq 0} \frac{1}{\left(h^{s}+\langle n \theta\rangle^{s}\right)\left(H^{s}+\left|n+\delta_{n}\right|^{s}\right)},
$$

где $H=h /\left|\theta-\theta^{\prime}\right|$ и для всех $n \neq 0$ имеем

$$
\left|\delta_{n}\right|=\frac{\left|m_{1}(n)-n \theta\right|}{\left|\theta-\theta^{\prime}\right|}<\frac{1}{2\left|\theta-\theta^{\prime}\right|} .
$$

Так как

$$
\sum_{n \neq 0} \frac{1}{h^{s}+\langle n \theta\rangle^{s}}\left|\frac{1}{|n|^{s}}-\frac{1}{H^{s}+\left|n+\delta_{n}\right|^{s}}\right| \leqslant C_{1}(\theta, s) \sum_{n \neq 0} \frac{1}{\langle n \theta\rangle^{s}|n|^{s+1}}=O(1),{ }^{1}
$$

получаем

$$
S_{2}=\frac{1}{\left|\theta-\theta^{\prime}\right|^{s}} \sum_{n \neq 0} \frac{1}{\left(h^{s}+\langle n \theta\rangle^{s}\right)|n|^{s}}+O(1)=\frac{2}{\left|\theta-\theta^{\prime}\right|^{s}} \sum_{n \geqslant 1} \frac{1}{\left(h^{s}+\langle n \theta\rangle^{s}\right) n^{s}}+O(1) .
$$

Аналогично доказывается, что

$$
S_{3}=\frac{2}{\left|\theta-\theta^{\prime}\right|^{s}} \sum_{n \geqslant 1} \frac{1}{\left(h^{s}+\left\langle n \theta^{\prime}\right\rangle^{s}\right) n^{s}}+O(1) .
$$

Таким образом, остается убедиться в том, что $S_{4}=O(1)$. Если $m \notin\left\{m_{1}(n), m_{2}(n)\right\}$, то

$$
|m-n \theta|>\frac{1}{2}, \quad\left|m-n \theta^{\prime}\right|>\frac{1}{2} .
$$

Поэтому

$$
S_{4} \leqslant \sum_{(m, n) \in \Omega} \frac{1}{|m-n \theta|^{s}\left|m-n \theta^{\prime}\right|^{s}}
$$

где $\Omega$ определяется неравенствами (2.1). Покажем, что последний ряд сходится. Для этого положим

$$
m-n \theta=k+\xi, \quad m-n \theta^{\prime}=l+\eta,
$$

где $k, l$ - целые числа и $|\xi|<1 / 2,|\eta|<1 / 2$. Если $(m, n) \in \Omega$, то $k \neq 0, l \neq 0$. Значит,

$$
\sum_{(m, n) \in \Omega} \frac{1}{|m-n \theta|^{s}\left|m-n \theta^{\prime}\right|^{s}}=\sum_{k \neq 0, l \neq 0} \sum_{(m, n) \in \Pi(k, l)} \frac{1}{|k+\xi|^{s}|l+\eta|^{s}}
$$

\footnotetext{
1Этот ряд сходится при любом $s>0$, что нетрудно установить применением преобразования Абеля и, например, первой из оценок (1.1).
} 


$$
\leqslant C_{2}(\theta) \sum_{k \neq 0, l \neq 0} \frac{1}{(|k|-1 / 2)^{s}(|l|-1 / 2)^{s}}<\infty
$$

(здесь $\Pi(k, l)$ - множество всех пар $(m, n) \in \Omega$, для которых имеют место равенства $(2.2)$ с данными $k, l$; ясно, что число таких пар ограничено некоторой константой $\left.C_{2}(\theta)\right)$.

Лемма доказана.

Пусть

$$
a \theta^{2}+b \theta+c=0
$$

где $a, b, c$ - целые взаимно простые числа, $a>0$. Модуль $\mathfrak{M}_{1}=a \mathfrak{M}=\{a,-a \theta\}$, подобный модулю $\mathfrak{M}$, содержится в своем кольце множителей $\mathfrak{D}=\{1, a \theta\}$ и поэтому для любого числа $\mu \in \mathfrak{M}_{1}$ его норма

$$
N_{\mathfrak{K} / \mathbb{Q}}(\mu)=\mu \mu^{\prime} \in \mathbb{Z} .
$$

Для произвольного натурального $c$ пусть $\mu_{j}=\mu_{j}(c) \in \mathfrak{M}_{1}, j=1, \ldots, Q(c),-$ некоторый фиксированный набор попарно неассоциированных решений норменного уравнения

$$
\left|N_{\mathfrak{K} / \mathbb{Q}}(\mu)\right|=c,
$$

где $\mu \in \mathfrak{M}_{1}$. Любое решение этого уравнения может быть однозначно представлено в виде

$$
\mu= \pm \mu_{j} \varepsilon^{k}, \quad j=1, \ldots, Q(c), \quad k \in \mathbb{Z},
$$

где $\varepsilon>1$ - основная единица порядка $\mathfrak{D}$.

Теорема. Если $\theta+\theta^{\prime} \in \mathbb{Z}$, то при $h \rightarrow 0$ справедлива оченка

$$
\sum_{n \geqslant 1} \frac{1}{\left(h^{s}+\langle n \theta\rangle^{s}\right) n^{s}}=\frac{a^{2 s}\left|\theta-\theta^{\prime}\right|^{s}}{\ln \varepsilon} \sum_{c \geqslant 1} \frac{Q(c)}{c^{s}} \ln h^{-1}+O(1) .
$$

ДокАзАтЕЛЬСтво. Очевидно, что

$$
S(h, \mathrm{M}, s)=a^{2 s} S\left(a h, \mathrm{M}_{1}, s\right),
$$

где $\mathrm{M}_{1}=a \mathrm{M}$ - решетка, соответствующая модулю $\mathfrak{M}_{1}$. Следуя методу, предложенному в [4], докажем при $h \rightarrow 0$ оценку

$$
S\left(h, \mathrm{M}_{1}, s\right)=\frac{4}{\ln \varepsilon} \sum_{c \geqslant 1} \frac{Q(c)}{c^{s}} \ln h^{-1}+O(1) .
$$

Тогда утверждение теоремы будет непосредственно следовать из этой оценки и леммы.

Доказательство оценки (2.5) разбивается на несколько этапов.

$A$. Сумму $S\left(h, \mathrm{M}_{1}, s\right)$ будем оценивать послойно, объединяя в слой те точки решетки $\mathrm{M}_{1}$, которые изображают решения уравнения (2.3) при фиксированном $c$. Поскольку $(x, y)=\left(\mu, \mu^{\prime}\right)$, имеем

$$
S\left(h, \mathrm{M}_{1}, s\right)=h^{-2 s} \sum_{0 \neq \mu \in \mathfrak{M}_{1}} \frac{1}{\left(1+\left|h^{-1} \mu\right|^{s}\right)\left(1+\left|h^{-1} \mu^{\prime}\right|^{s}\right)}=2 h^{-2 s} \sum_{c \geqslant 1} \sum_{j=1}^{Q(c)} \sum_{k \in \mathbb{Z}} F(k),
$$

где введены обозначения

$$
F(t)=\frac{1}{\left(1+z^{s}\right)\left(1+(\omega / z)^{s}\right)}, \quad z=h^{-1}\left|\mu_{j}\right| \varepsilon^{t}, \quad \omega=h^{-2} c .
$$

Будем считать $0<h<1$, так что $\omega>1$. Положим

$$
\Sigma=\sum_{k \in \mathbb{Z}} F(k), \quad I=\int_{\mathbb{R}} F(t) d t
$$

и пусть $\delta=\Sigma-I$. 
Б. Вычислим интеграл $I$, сделав замену переменой $t \rightarrow z$ :

$$
\begin{aligned}
I & =\frac{1}{\ln \varepsilon} \int_{\mathbb{R}_{+}} \frac{1}{\left(1+z^{s}\right)\left(1+(\omega / z)^{s}\right)} \cdot \frac{d z}{z}=\frac{1}{\ln \varepsilon} \int_{\mathbb{R}_{+}} \frac{z^{s-1}}{\left(1+z^{s}\right)\left(z^{s}+\omega^{s}\right)} d z \\
& =\frac{1}{\ln \varepsilon} \cdot \frac{\ln \omega}{\omega^{s}-1}=\frac{1}{\ln \varepsilon} \omega^{-s}\left(\ln \omega+C_{3}(\omega)\right),
\end{aligned}
$$

где величина

$$
C_{3}(\omega)=\frac{\ln \omega}{\omega^{s}-1}<\frac{\ln \omega}{\omega-1}<1 .
$$

B. Оценим погрешность $\delta$, используя очевидное неравенство

$$
|\delta| \leqslant \int_{\mathbb{R}}\left|F^{\prime}(t)\right| d t
$$

Нетрудно проверить, что

$$
F^{\prime}(t)=-s \ln \varepsilon \frac{z^{s}-(\omega / z)^{s}}{\left[\left(1+z^{s}\right)\left(1+(\omega / z)^{s}\right)\right]^{2}},
$$

поэтому

$$
\begin{aligned}
\int_{\mathbb{R}}\left|F^{\prime}(t)\right| d t & =s \int_{\mathbb{R}_{+}} \frac{\left|z^{s}-(\omega / z)^{s}\right|}{\left[\left(1+z^{s}\right)\left(1+(\omega / z)^{s}\right)\right]^{2}} \cdot \frac{d z}{z} \\
& \leqslant s \int_{\mathbb{R}_{+}} \frac{z^{s}}{\left[\left(1+z^{s}\right)\left(1+(\omega / z)^{s}\right)\right]^{2}} \cdot \frac{d z}{z}+s \int_{\mathbb{R}_{+}} \frac{(\omega / z)^{s}}{\left[\left(1+z^{s}\right)\left(1+(\omega / z)^{s}\right)\right]^{2}} \cdot \frac{d z}{z} \\
& =2 s \int_{\mathbb{R}_{+}} \frac{(\omega / z)^{s}}{\left[\left(1+z^{s}\right)\left(1+(\omega / z)^{s}\right)\right]^{2}} \cdot \frac{d z}{z} \leqslant 2 s \int_{\mathbb{R}_{+}} \frac{(\omega / z)^{-s}}{\left(1+z^{s}\right)^{2}} \cdot \frac{d z}{z}=2 \omega^{-s} .
\end{aligned}
$$

Следовательно, справедливо неравенство

$$
|\delta| \leqslant 2 \omega^{-s}
$$

Г. Применим полученные выше оценки, предварительно переписав их в терминах $h$ и $c$. Имеем

$$
\omega^{-s}=h^{2 s} c^{-s}, \quad \ln \omega=2 \ln h^{-1}+\ln c,
$$

поэтому

$$
\begin{aligned}
S\left(h, \mathrm{M}_{1}, s\right) & =2 h^{-2 s} \sum_{c \geqslant 1} \sum_{j=1}^{Q(c)}(I+\delta)=2 h^{-2 s} \sum_{c \geqslant 1} \sum_{j=1}^{Q(c)} I+2 h^{-2 s} \sum_{c \geqslant 1} \sum_{j=1}^{Q(c)} \delta \\
& =\frac{2}{\ln \varepsilon} \sum_{c \geqslant 1} \sum_{j=1}^{Q(c)} \frac{2 \ln h^{-1}+\ln c+C_{3}(\omega)}{c^{s}}+2 \sum_{c \geqslant 1} \sum_{j=1}^{Q(c)} h^{-2 s} \delta \\
& =\frac{4}{\ln \varepsilon} \sum_{c \geqslant 1} \frac{Q(c)}{c^{s}} \ln h^{-1}+\Delta_{1}+\Delta_{2},
\end{aligned}
$$

где величины

$$
\Delta_{1}=\frac{2}{\ln \varepsilon} \sum_{c \geqslant 1} \frac{Q(c)\left(\ln c+C_{3}(\omega)\right)}{c^{s}}, \quad \Delta_{2}=2 \sum_{c \geqslant 1} \sum_{j=1}^{Q(c)} h^{-2 s} \delta .
$$

Ясно, что $\Delta_{1}=O(1)$, а так как $\left|h^{-2 s} \delta\right| \leqslant 2 c^{-s}$, то и $\Delta_{2}=O(1)$. Тем самым оценка $(2.5)$ установлена, а теорема полностью доказана. 
ЗАмечаниЕ. Если поле $\mathfrak{K}$ одноклассно, а модуль $\mathfrak{M}$ есть кольцо целых чисел этого поля, то участвующий в формуле (2.4) ряд Дирихле представляет собой дзета-функцию Дедекинда $\zeta_{\mathfrak{K}}(s)$ поля $\mathfrak{K}$.

СледствиЕ. Если $\theta+\theta^{\prime} \in \mathbb{Z}$, то при $N \rightarrow \infty$ справедлива оценка

$$
\sum_{n=1}^{N} \frac{1}{\left(N^{1-s}+\langle n \theta\rangle^{s}\right) n^{s}}=\frac{a^{2 s}\left|\theta-\theta^{\prime}\right|^{s}}{\ln \varepsilon} \sum_{c \geqslant 1} \frac{Q(c)}{c^{s}} \nu \ln N+O(1),
$$

где $\nu=1-1 / s$.

ДокАЗАТЕЛЬСтво. Достаточно положить $h=N^{-\nu}$ в формуле (2.4) и заметить, что

$$
\sum_{n>N} \frac{1}{\left(N^{1-s}+\langle n \theta\rangle^{s}\right) n^{s}}<N^{s-1} \sum_{n>N} \frac{1}{n^{s}}=O(1) .
$$

\section{СПИСОК ЦИТИРОВАННОЙ ЛИТЕРАТУРЫ}

[1] В. Шмидт, Диофантовы приближения, Мир, М., 1983. [2] А.H. Kruse, Acta Arith., 12 (1967), 229-261. [3] S. Haber, C. Osgood, Pacific J. Math., 31 (1969), 383-394. [4] Н. Н. Осипов, Вычислительные технологии, 11 (спецвыпуск) (2006), 81-89. [5] З. И. Боревич, И. Р. Шафаревич, Теория чисел, Наука, М., 1985.

\section{Н.Н. Осипов}

Политехнический институт Сибирского федерального университета, г. Красноярск E-mail: nnosipov@rambler.ru
Поступило 09.02.2007

Исправленный вариант 13.06.2007 